\title{
Chemical reaction and Newtonian heating effects on steady convection flow of a micropolar fluid with second order slip at the boundary
}

\author{
M. Kamran ${ }^{1}$, Benchawan Wiwatanapataphee \\ Department of Mathematics and Statistics \\ Curtin University of Technology, \\ GPO Box U1987, Perth \\ WA 6845 Australia
}

\begin{abstract}
In this paper, asymptotic analysis of the chemical reaction and the Newtonian heating parameters is carried out. A mathematical model of a convective micropolar fluid flow over a permeable stretching/shrinking sheet is taken into account in the presence of the slip flow regime. A nonlinear system of transformed equations is solved by a semi-analytical technique called Homotopy Analysis Method (HAM). The current investigation is in a good agreement with the already published analytical and the numerical results with the help of tabular and graphical representations. In comparison with the stretching sheet, it is observed that the shrinking sheet produces a wider concentration boundary layer thickness by a small change in the chemical reaction parameter. In contrast to the stretching sheet, the Newtonian heating parameter raises the thermal boundary layer thickness by $39.93 \%$ for the shrinking sheet. The chemical reaction with the Newtonian heating effect is an important consideration in the solidification process of the liquid crystals and the polymeric suspensions.
\end{abstract}

Keywords: micropolar fluid; stretching/shrinking sheet; slip flow condition; Newtonian heating; chemical reaction

\section{Nomenclature}

$A$ constant coefficient for mean free path, $m$

$B$ square of constant coefficient for mean free path, $m^{2}$

$c_{p}$ specific heat at constant pressure, $J K^{-1} K^{-1}$

$C_{f}$ local skin friction coefficient

$C$ concentration of the species

$C_{m}$ dimensionless Chemical reaction parameter

$D_{m}$ mass diffusivity

$d$ mean free path, $m$

$f$ dimensionless velocity $f_{w}$ dimensionless suction/injuction parameter

$g$ gravitational acceleration, $m s^{-2}$

$G r_{x}$ dimensionless local Grashof number

$h_{s}$ surface heat transfer coefficient, $W m^{-2} K^{-1}$

$h$ dimensionless micro rotational velocity

$j$ microinertia per unit mass,

$K$ dimensionless material parameter

$\xi$ first order homogeneous constant reaction rate

$k$ thermal conductivity, $W m^{-1} K^{-1}$

$k_{n}$ Knudsen number

\footnotetext{
${ }^{1}$ Correspondence to: M.Kamran

E-mail: muhammad.kamran@curtin.edu.au
} 
$M_{x}$ local wall couple stress

$N u$ local Nusselt number

$\operatorname{Pr}$ dimensionless Prandtl number

$R e_{x}$ dimensionless local Reynolds number

Sc dimensionless Schmidt number

$S h_{x}$ dimensionless Sherwood number

$s$ stretching/ shrinking constant

$T$ fluid temperature, $K$

$u$ horizontal velocity component, $\mathrm{ms}^{-1}$

$v$ vertical velocity component, $m s^{-1}$

$v_{o}$ suction/injection velocity, $m s^{-1}$

$x$ horizontal Direction, $m$

$y$ transverse directions, $m$

\section{Greek symbols}

$\alpha$ dimensionless first order slip flow parameter

$\beta$ dimensionless second order slip flow parameter

$\beta_{T}$ coefficient of thermal expansion, $K^{-1}$ $\beta_{c}$ coefficient of thermal expansion with concentration

$\gamma$ spin gradient viscosity coefficient, $\mathrm{Kgms}^{-1}$

$\delta$ dimensionless Newtonian heating parameter

$\epsilon$ momentum accommodation coefficient

$\theta$ dimensionless temperature

$\lambda$ dimensionless Bouncy parameter

$\Lambda$ dimensionless thermal to bouncy ratio parameter

$\mu$ dynamic viscosity, $\mathrm{Nsm}^{-2}$

$\nu$ kinematic viscosity, $m^{2} s^{-1}$

$\psi$ stream function, $m^{2} s^{-1}$

$\rho$ fluid density, $\mathrm{Kgm}^{-3}$

$\chi$ microrotation viscosity, $\mathrm{Kgm}^{-1} \mathrm{~s}^{-1}$

$\omega$ micro rotational velocity (cartesian), $\mathrm{ms}^{-1}$

\section{Subscripts}

$w$ wall

$\infty$ free stream.

\section{Introduction}

It is well known that theories of classical fluid mechanics could not adequately explain the particular characteristics of polymeric fluids, fluids with additives or the animal blood. Such fluids exhibit the micro-rotational effects and micro-rotational inertia, and later they named as micropolar fluids in the theory of Eringen [1]. It describes the effects of micro-structured particles with the help of an additional transport equation by representing the local angular momentum, and in addition to some extra constitutive parameters, those are not reported in the classical Navier-Stokes theory of fluid mechanics. Later on, this theory extended to some basic viscous micropolar fluid flows such as Couette and Poiseuille flow [2], heat conducting micropolar fluid flows [3, 4] and to investigate the boundary layer [5] micropolar fluid flow. An experimental study by Ariman et al. [6] has established a good comparison with the exact solution of a steady and pulsatile micropolar fluid flow through a circular tube. Migun [7] proposed an experimental method to determine parameters which characterize the micro-particles of the micropolar fluid. The volumetric flow rate of the micropolar fluid has been examined when it passes through the two circular cylinders of different cross section and the obtained results are good in agreement with the already published results. Kolpashchikov et al. [8] experimentally calculated the viscosity coefficients of the micropolar fluid flow with the help of specific viscometric and thermal measurements along with the concrete form of the boundary conditions for the microrotation. They quantitatively examined the governing flow parameters of the steady state micropolar fluid flow through any constant cross section of the capillary tube.

In last few decades, the study of heat and mass transfer in micropolar fluids has received significant attention due to their applications in various industrial processes such as oil extraction, solidification of liquid crystals, polymeric suspensions, etc. So far, many investigations of the micropolar fluid flow have been reported under different kinds of conditions. For example, Eldabe and Ouaf [9] numerically investigated the heat and mass transfer magnetohydrodynamics (MHD) micropolar fluid flow over a linear stretching surface in the presence of viscous dissipation and the Joule heating. They found that an increase in the viscous dissipation and the Joule heating causes to raise the micropolar fluid temperature. Ishak et al. [10] studied the boundary layer micropolar fluid flow over a moving and fixed porous surface with the dilute concentration of microelements. They investigated one of the cases in which plate and free stream fluid flow move in opposite direction, and the dual solutions have been reported. Ishak [11] reported a numerical solution of an incompressible radiating micropolar fluid flow over a stretching sheet. Ishak reported that the thermal boundary layer thickness increases with the increasing value of the 
radiation parameter. Bachok et al. [12] examined the unsteady thermal boundary layer flow of an incompressible micropolar fluid over an impulsively stretching sheet. Zheng et al. [13] reported the dual solutions of the heat transfer micropolar fluid flow past over a stretching/shrinking sheet with the condition of power-law surface velocity and the temperature distribution. They investigated that both solutions are physically meaningful and closely related to each other. An investigation of magneto-micropolar fluid flow through a pipe has been investigated by Sherief et al. [14]. An exact analytical investigation of magneto-micropolar fluid flow has been reported by Ezzat and Shreen [15]. Chaudhary and Jain [16] investigated the slip effect on the heat and mass transfer MHD radiative micropolar fluid flow past over a vertical porous plate which is present in the porous media.

In the study of heat and mass transfer, many reactions involve the simultaneous diffusion of molecular species. These species appear or disappear through a chemical reaction either within or at the boundary of the phase. Categorically, there are two types of chemical reactions, the one is uniform throughout a given phase named as the homogeneous chemical reaction and the second one takes place within or at the boundary of the phase of interest, called heterogeneous chemical reaction. The presence of chemical reaction is greatly important in some industrial processes such as polymer production, to design the chemical processing equipment, for example, to design the machine which is used to spray on the agriculture fields, food processing, the manufacturing of ceramics or glassware, dying in cloth industry and the cooling of the tower. The effect of chemical reaction on magnetomicropolar fluid flow over a porous plate in the porous medium has been analyzed by the Mohamed and Dahab [17]. In their analysis, the influence of thermal radiation and heat generation has also been conducted. Chamkha et al. [18] studied the influence of the chemical reaction on the radiating magneto-micropolar fluid flow over a vertical heated plate with the ohmic heating. It is observed that with these parameters, an increase in the chemical reaction causes to a decrease in the concentration profile and its boundary layer thickness. A numerical investigation of chemical reaction on MHD free convective heat and mass transfer micropolar fluid flow past over an oscillatory plate in the rotating frame of reference has been investigated by Bakr [19]. Hayat et al. [20] reported a study of the chemical reaction on mixed convective radiating magneto-micropolar fluid flow past over a stretching sheet. Pal et al. [21] analysed a combined study of the chemical reaction and the Hall effects on the thermally radiating micropolar fluid flow past over a semi-infinite vertical impermeable plate.

In the analysis of the boundary layer of the viscous fluids, it is well known that the velocity of fluid particles matches with the velocity of the solid surface. This effect is known as a no-slip effect. On the other hand, some fluid particles no longer take the velocity of the solid surface and slip along the surface due to their finite tangential velocity. This effect can't be ignored and resulting flow behaviour is called as a slip-flow regime. In heat and mass transfer, the slip flow effect has important engineering applications such as transmission lines, refrigerating coils, polishing artificial heart valves and internal cavities. A mathematical model [22] for slip flow regime has an advantage over the other reported slip flow models such as $1^{\text {st }}$ order, $1.5^{\text {th }}$ order or $2^{\text {nd }}$ order slip flow models (see references in [22]). This slip flow model has a very close agreement to the solutions computed for the whole range of the Knudsen number. Moreover, Wu's [22] slip flow model has been applied to the quiescent fluid [23, 24, 25, 26] and furthermore, extended to the micropolar fluid flows [27, 28, 29] under different type of parametric flow behaviours and boundary conditions.

The objective of this study is to present the semi-analytical results for the micropolar fluid flow past over a stretching/shrinking permeable sheet with the second order slip flow model reported by $\mathrm{Wu}$ [22]. The first order homogeneous chemical reaction and the Newtonian heating effects are taken into account. In this heat and mass transfer flow, the detailed asymptotic analysis for the involved parameters is assumed to be described, and it would be valid for the analytically exact and numerical results under special cases. Therefore, the presentation of this study is organized as follows. In section 2 , the governing equations with the pertaining boundary conditions are described. It is shown that the flow model is reduced to the coupled nonlinear set of ordinary differential equations. Semi-analytical series solutions of the transformed set of equations are presented in section 3. Under particular cases, the asymptotic analysis of the current study is validated in section 4 with the help of the already published analytical and numerical results. Section 5 consists of the results and discussion part of all the involved parameters. Furthermore, a concluding remark is presented in section 6 .

\section{Governing Equations}

Considered a steady state mixed convective incompressible micropolar fluid flow past over a porous surface which stretches and shrinks with the velocity $u=s u_{w}(x)$, where $u_{w}(x)=a x$ and $a$ is a positive dimensional constant, along the axis of flow. The flow is taken along the $x$-axis and $y$-axis normal to it. Wu's [22] second order slip flow model $\left(u_{\text {slip }}\right)$ and the Newtonian heating [30] are assumed in the boundary conditions. With the help of usual Boussinesq approximation, the mathematical form of governing equations [20, 27] can be written as 


$$
\begin{aligned}
\frac{\partial u}{\partial x}+\frac{\partial v}{\partial y} & =0 \\
u \frac{\partial u}{\partial x}+v \frac{\partial u}{\partial y} & =\frac{\mu+\chi}{\rho}\left(\frac{\partial^{2} u}{\partial y^{2}}\right)+\frac{\chi}{\rho}\left(\frac{\partial \omega}{\partial y}\right)+\mathrm{g} \beta_{T}\left(T-T_{\infty}\right)+\mathrm{g} \beta_{C}\left(C-C_{\infty}\right) \\
u \frac{\partial \omega}{\partial x}+v \frac{\partial \omega}{\partial y} & =\frac{\gamma}{\rho j}\left(\frac{\partial^{2} \omega}{\partial y^{2}}\right)-\frac{\chi}{\rho j}\left(2 \omega+\frac{\partial u}{\partial y}\right) \\
u \frac{\partial T}{\partial x}+v \frac{\partial T}{\partial y} & =\frac{k}{\rho c_{p}}\left(\frac{\partial^{2} T}{\partial y^{2}}\right) \\
u \frac{\partial C}{\partial x}+v \frac{\partial C}{\partial y} & =D_{m}\left(\frac{\partial^{2} C}{\partial y^{2}}\right)-\xi\left(C-C_{\infty}\right)
\end{aligned}
$$

with boundary conditions

$$
\left.\begin{array}{r}
u=s u_{w}(x)+u_{\text {slip }} ; v=v_{o} ; \omega=-n \frac{\partial u}{\partial y} ; k \frac{\partial T}{\partial y}=-h_{s} T ; C=C_{w} \text { at } y=0, \\
u \rightarrow 0 ; \omega \rightarrow 0 ; T \rightarrow T_{\infty} ; C \rightarrow C_{\infty} ; \text { as } y \rightarrow \infty .
\end{array}\right\}
$$

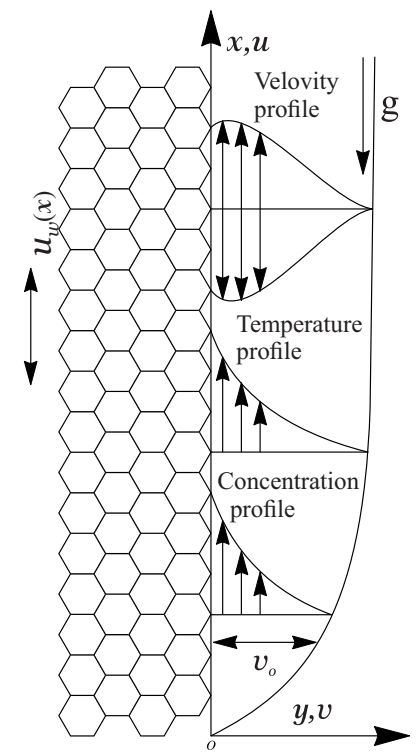

Figure 1 Flow model.

The spin gradient viscosity is considered as $\gamma=(\mu+\chi / 2) j=\mu(1+K / 2) j$, where $K=\chi / \mu$ and $j=\nu / a$ are the material parameter and micro inertia density [31], respectively. Moreover, $v_{o}>0$ and $v_{o}<0$ represent the suction and injection velocities of the permeable sheet, respectively. The constant $n$ in Eq. (6) $)_{3}$ can be chosen from $0 \leq n \leq 1$, in which([32] and see the references therein) $n=0$ corresponds to the strong concentration of micro-elements in the micropolar fluid flow near the sheet which are unable to rotate $\omega=0$, and $n=1$ signifies the turbulent flow. However, $n=0.5$ represents the vanishing of anti-symmetric stress tensor which corresponds to the dilute micropolar fluid and this dilute fluid is considered in the present study. Furthermore, the $u_{\text {slip }}$ is slip velocity of the stretching $(s>0)$ /shrinking $(s<0)$ sheet, which is given by $\mathrm{Wu}[22]$ as

$$
u_{\text {slip }}=\frac{2}{3}\left(\frac{3-\epsilon l^{3}}{\epsilon}-\frac{3}{2} \frac{1-l^{2}}{k_{n}}\right) d \frac{\partial u}{\partial y}-\frac{1}{4}\left(l^{4}+\frac{2}{k_{n}^{2}}\left(1-l^{2}\right)\right) d^{2} \frac{\partial^{2} u}{\partial y^{2}}=A \frac{\partial u}{\partial y}+B \frac{\partial^{2} u}{\partial y^{2}},
$$

where $l$ is chosen from $\min \left[1 / k_{n}, 1\right]$ which leads to $0<l \leqslant 1$ for any value of the Knudsen $\left(k_{n}\right)$ number, and $\epsilon$ is the momentum accommodation coefficient which is defined as $0<\epsilon \leqslant 1$. Furthermore, the molecular mean free path 
$d$ is always positive and $B$ is consequently negative in magnitude. However, by introducing the non-dimensional similarity variables with velocity components $u=a x f^{\prime}(\eta)$ and $v=-(a \nu)^{1 / 2} f(\eta)$ as follows:

$$
\eta=\sqrt{a / \nu} y, \omega=\sqrt{a / \nu} a x h(\eta), \theta(\eta)=\frac{T-T_{\infty}}{T_{\infty}}, \phi=\frac{C-C_{\infty}}{C_{w}-C_{\infty}},
$$

and the governing equations (Eqs. (2)-(6)) are transformed into a set of non-linear ordinary differential equations as follows:

$$
\begin{aligned}
(1+K) f^{\prime \prime \prime}-\left(f^{\prime}\right)^{2}+f f^{\prime \prime}+K h^{\prime}+\lambda \theta+\lambda \Lambda \phi & =0 \\
(1+K / 2) h^{\prime \prime}-f^{\prime} h+f h^{\prime}-K\left(2 h+f^{\prime \prime}\right) & =0 \\
\theta^{\prime \prime}+\operatorname{Pr} f \theta^{\prime} & =0 \\
\phi^{\prime \prime}+S c f \phi^{\prime}-C_{m} S c \phi & =0
\end{aligned}
$$

with the boundary conditions

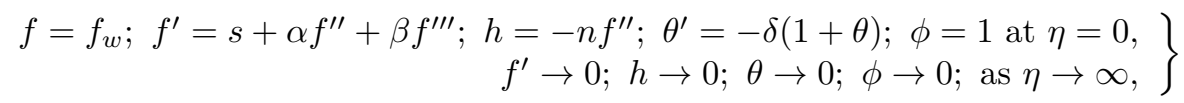

where prime $\left({ }^{\prime}\right)$ represents the derivative with respect to the $\eta$. The non-dimensional physical parameters used in Eqs. (7)-(10) are bouncy parameter $\lambda=G r_{x} / R e_{x}$ for the local Grashof number $G r_{x}=\mathrm{g} \beta_{T} T_{\infty} x / a \nu$ and the local Reynolds number $R e_{x}=a x^{2} / \nu$, concentration to thermal bouncy ratio parameter $\Lambda=\beta_{c}\left(C_{w}-C_{\infty}\right) / \beta_{T} T_{\infty}$, Prandtl number $\operatorname{Pr}=\mu c_{p} / k$, Schmidt number $S c=\nu / D_{m}$ and chemical reaction parameter $C_{m}=\xi / a$. In Eq. (11), suction or injection parameter $f_{w}=-(a \nu)^{-\frac{1}{2}} v_{o}$, first order slip flow parameter $\alpha=A \sqrt{a / \nu}>0$, second order slip flow parameter $\beta=B a / \nu<0$, Newtonian heating parameter $\delta=\frac{h_{s}}{k} \sqrt{\nu / a}$ are defined.

The physical quantities including local skin friction $\left(C_{f_{x}}\right)$, local wall couple stress $\left(M_{x}\right)$, the local Nusselt number $\left(N u_{x}\right)$ and the local Sherwood number $\left(S h_{x}\right)$ are determined as follows:

$$
\begin{aligned}
C_{f_{x}} & =\frac{-\tau_{w}}{\rho(a x)^{2}}, \text { for } \tau_{w_{x}}=(\mu+\chi) \frac{\partial u}{\partial y}+\left.\chi \omega\right|_{y=0}, \\
M_{x} & =\frac{-m_{w}}{\rho x(a x)^{2}}, \text { for } m_{w}=\left.\left(\mu+\frac{\chi}{2}\right) j \frac{\partial \omega}{\partial y}\right|_{y=0}, \\
N u_{x} & =\frac{-x q_{w}}{\left(T-T_{\infty}\right)}, \text { for } q_{w}=\left.\frac{\partial T}{\partial y}\right|_{y=0}, \\
S h_{x} & =\frac{-x S_{m}}{\left(C-C_{w}\right)}, \text { for } S_{m}=\left.\frac{\partial C}{\partial y}\right|_{y=0}
\end{aligned}
$$

Similarity transformation yields

$$
\begin{aligned}
C_{f_{x}} R e_{x}^{\frac{1}{2}} & =-\left.(1+K-n K) f^{\prime \prime}(\eta)\right|_{\eta=0}, \\
M_{x} R e_{x} & =-\left.\left(1+\frac{K}{2}\right) h^{\prime}(\eta)\right|_{\eta=0}, \\
N u_{x} R e_{x}^{-\frac{1}{2}} & =\left.\delta\left(1+\frac{1}{\theta(\eta)}\right)\right|_{\eta=0}, \\
S h_{x} R e_{x}^{-\frac{1}{2}} & =-\left.\phi^{\prime}(\eta)\right|_{\eta=0} .
\end{aligned}
$$

\section{Solution by Homotopy Analysis Method}

Homotopy Analysis Method (HAM) developed by Liao [33, 34] producing approximately analytical series solution of the nonlinear ordinary differential equations has an advantage on the perturbation and non-perturbation 
methods and has a great freedom to adjust the convergence of the series solution. Here, HAM is applied to solve the system of coupled nonlinear ODEs (Eqs. (7)-(10)) with its pertaining boundary conditions (Eq. (11)). The homotopy series solutions of the $f(\eta), h(\eta), \theta(\eta)$ and the $\phi(\eta)$ with the help of a set of the base functions $\left\{\eta^{r} \exp (-i \eta) \mid r \geq 0, i \geq 0\right\}$ can be written as

$$
\left.\begin{array}{l}
f(\eta)=\sum_{r=0}^{\infty} \sum_{i=0}^{\infty} a_{r, i} \eta^{r} \exp (-i \eta), \\
h(\eta)=\sum_{r=0}^{\infty} \sum_{i=0}^{\infty} b_{r, i} \eta^{r} \exp (-i \eta), \\
\theta(\eta)=\sum_{r=0}^{\infty} \sum_{i=0}^{\infty} c_{r, i} \eta^{r} \exp (-i \eta), \\
\phi(\eta)=\sum_{r=0}^{\infty} \sum_{i=0}^{\infty} d_{r, i} \eta^{r} \exp (-i \eta),
\end{array}\right\}
$$

where $a_{r, i}, b_{r, i} c_{r, i}$ and $d_{r, i}$ are constants.

\subsection{Zero-order deformation equations}

The first rule of the solution expressions represented by Eq. (16) and the boundary conditions (in Eq. (11)) play an important role to choose the initial solutions as

$$
\left.\begin{array}{rl}
f_{0}(\eta) & =f_{w}+\frac{s\left(1-e^{-\eta}\right)}{1+\alpha-\beta}, \\
h_{0}(\eta) & =\frac{s n e^{-\eta}}{1+\alpha-\beta}, \\
\theta_{0}(\eta) & =\frac{\delta e^{-\eta}}{1-\delta} ; \delta \neq 1, \\
\phi_{0}(\eta) & =e^{-\eta},
\end{array}\right\}
$$

with the corresponding linear operators

$$
\mathcal{L}_{f}=\frac{\partial^{3}}{\partial \eta^{3}}-\frac{\partial}{\partial \eta}, \mathcal{L}_{h}=\frac{\partial^{2}}{\partial \eta^{2}}+\frac{\partial}{\partial \eta}, \mathcal{L}_{\theta}=\frac{\partial^{2}}{\partial \eta^{2}}+\frac{\partial}{\partial \eta}, \text { and } \mathcal{L}_{\phi}=\frac{\partial^{2}}{\partial \eta^{2}}+\frac{\partial}{\partial \eta}
$$

which satisfy the following properties

$$
\left.\begin{array}{rl}
\mathcal{L}_{f}\left[X_{1}+X_{2} e^{\eta}+X_{3} e^{-\eta}\right] & =0, \\
\mathcal{L}_{h}\left[X_{4}+X_{5} e^{-\eta}\right] & =0, \\
\mathcal{L}_{\theta}\left[X_{6}+X_{7} e^{-\eta}\right] & =0 \\
\mathcal{L}_{\phi}\left[X_{8}+X_{9} e^{-\eta}\right] & =0
\end{array}\right\}
$$

in which $X_{i}(i$ varies from 1 to 9$)$ are arbitrary constants. If $q(\in[0,1])$ represents the embedding parameter, $\hbar$ denotes the non-zero auxiliary parameter and $H(\eta)$ is taken as a non-zero auxiliary function then, in Homotopy Analysis Method, continuous mappings are defined as $f(\eta) \rightarrow \psi(\eta ; q), h(\eta) \rightarrow \varphi(\eta ; q), \theta(\eta) \rightarrow \Phi(\eta ; q), \phi(\eta) \rightarrow$ $\Psi(\eta ; q)$ such that, when embedding parameter $(q)$ changes from 0 to 1 then $\{\psi(\eta ; q), \varphi(\eta ; q), \Phi(\eta ; q), \Psi(\eta ; q)\}$ vary from initial solutions $\left\{f_{0}(\eta), h_{0}(\eta), \theta_{0}(\eta), \phi_{0}(\eta)\right\}$ to the exact solutions $\{f(\eta), h(\eta), \theta(\eta), \phi(\eta)\}$. In order to ensure it, the nonlinear operators with their corresponding unknown functions, respectively, are defined as

$$
\begin{aligned}
& \mathcal{N}_{f}[\psi(\eta ; q)]=(1+K) \psi(\eta ; q)^{\prime \prime \prime}-\left(\psi(\eta ; q)^{\prime}\right)^{2}+\psi(\eta ; q) \psi(\eta ; q)^{\prime \prime}+K \varphi(\eta ; q)^{\prime}+\lambda \Phi(\eta ; q)+\lambda \Lambda \Psi(\eta ; q), \\
& \mathcal{N}_{h}[\varphi(\eta ; q)]=(1+K / 2) \varphi(\eta ; q)^{\prime \prime}-\psi(\eta ; q)^{\prime} \varphi(\eta ; q)+\psi(\eta ; q) \varphi(\eta ; q)^{\prime}-K\left(2 \varphi(\eta ; q)+\psi(\eta ; q)^{\prime \prime}\right), \\
& \mathcal{N}_{\theta}[\Phi(\eta ; q)]=\Phi(\eta ; q)^{\prime \prime}+\operatorname{Pr} \psi(\eta ; q) \Phi(\eta ; q)^{\prime}, \\
& \mathcal{N}_{\phi}[\Psi(\eta ; q)]=\Psi(\eta ; q)^{\prime \prime}+\operatorname{Sc} \psi(\eta ; q) \Psi(\eta ; q)^{\prime}-C_{m} \operatorname{Sc} \Psi(\eta ; q),
\end{aligned}
$$


then the zero-order deformation equations can be written as

$$
\left.\begin{array}{rl}
(1-q) \mathcal{L}_{f}\left[\psi(\eta ; q)-f_{0}(\eta)\right] & =q \hbar_{f} H_{f}(\eta) \mathcal{N}_{f}[\psi(\eta ; q), \varphi(\eta ; q), \Phi(\eta ; q), \Psi(\eta ; q)], \\
(1-q) \mathcal{L}_{h}\left[\varphi(\eta ; q)-h_{0}(\eta)\right] & =q \hbar_{h} H_{h}(\eta) \mathcal{N}_{h}[\psi(\eta ; q), \varphi(\eta ; q), \Phi(\eta ; q), \Psi(\eta ; q)], \\
(1-q) \mathcal{L}_{\theta}\left[\Phi(\eta ; q)-\theta_{0}(\eta)\right] & =q \hbar_{\theta} H_{\theta}(\eta) \mathcal{N}_{\theta}[\psi(\eta ; q), \varphi(\eta ; q), \Phi(\eta ; q), \Psi(\eta ; q)], \\
(1-q) \mathcal{L}_{\phi}\left[\Psi(\eta ; q)-\phi_{0}(\eta)\right] & =q \hbar_{\phi} H_{\phi}(\eta) \mathcal{N}_{\phi}[\psi(\eta ; q), \varphi(\eta ; q), \Phi(\eta ; q), \Psi(\eta ; q)],
\end{array}\right\}
$$

with the following associated boundary conditions at $\eta=0$ and $\eta \rightarrow \infty$ as, i.e.

$$
\left.\begin{array}{l}
\psi(0 ; q)=f_{w},\left.\frac{\partial \psi(\eta ; q)}{\partial \eta}\right|_{\eta=0}=s+\left.\alpha \frac{\partial^{2} \psi(\eta ; q)}{\partial \eta^{2}}\right|_{\eta=0}+\left.\beta \frac{\partial^{3} \psi(\eta ; q)}{\partial \eta^{3}}\right|_{\eta=0},\left.\frac{\partial \psi(\eta ; q)}{\partial \eta}\right|_{\eta \rightarrow \infty} \rightarrow 0, \varphi(\infty ; q) \rightarrow 0, \\
\varphi(0 ; q)=-\left.n \frac{\partial^{2} \psi(\eta ; q)}{\partial \eta^{2}}\right|_{\eta=0},\left.\frac{\partial \Phi(\eta ; q)}{\partial \eta}\right|_{\eta=0}=-\delta(1+\Phi(0 ; q)), \Phi(\infty ; q) \rightarrow 0, \Psi(0 ; q)=1, \Psi(\infty ; q) \rightarrow 0 .
\end{array}\right\}
$$

It is obvious to show that the solutions of Eq. (20) subject to Eq. (21) when $q=0$ are

$$
\left.\begin{array}{l}
\psi(\eta ; 0)=f_{0}(\eta), \\
\varphi(\eta ; 0)=h_{0}(\eta), \\
\Phi(\eta ; 0)=\theta_{0}(\eta), \\
\Psi(\eta ; 0)=\phi_{0}(\eta) .
\end{array}\right\}
$$

Since $\hbar \neq 0$ and $H(\eta) \neq 0$, Eq. (20) and Eq. (21) are then equivalent to the Eqs. (7)-(10) with boundary conditions in Eq. (11) at $q=1$. We obtain

$$
\left.\begin{array}{c}
\psi(\eta ; 1)=f(\eta), \\
\varphi(\eta ; 1)=h(\eta), \\
\Phi(\eta ; 1)=\theta(\eta), \\
\Psi(\eta ; 1)=\phi(\eta) .
\end{array}\right\}
$$

By Taylor's theorem, $\psi(\eta ; q), \varphi(\eta ; q), \Phi(\eta ; q)$ and $\Psi(\eta ; q)$ can be expressed in the power series of $q$ as follows:

$$
\left.\begin{array}{l}
\psi(\eta ; q)=f_{0}(\eta)+\sum_{p=1}^{\infty} f_{p}(\eta) q^{p}, \\
\varphi(\eta ; q)=h_{0}(\eta)+\sum_{p=1}^{\infty} h_{p}(\eta) q^{p}, \\
\Phi(\eta ; q)=\theta_{0}(\eta)+\sum_{p=1}^{\infty} \theta_{p}(\eta) q^{p}, \\
\Psi(\eta ; q)=\phi_{0}(\eta)+\sum_{p=1}^{\infty} \phi_{p}(\eta) q^{p},
\end{array}\right\}
$$

in which

$$
f_{p}(\eta)=\left.\frac{1}{p !} \frac{\partial^{p} \psi(\eta, q)}{\partial q^{p}}\right|_{q=0}, h_{p}(\eta)=\left.\frac{1}{p !} \frac{\partial^{p} \varphi(\eta, q)}{\partial q^{p}}\right|_{q=0}, \theta_{p}(\eta)=\left.\frac{1}{p !} \frac{\partial^{p} \Phi(\eta, q)}{\partial q^{p}}\right|_{q=0}, \phi_{p}(\eta)=\left.\frac{1}{p !} \frac{\partial^{p} \Psi(\eta, q)}{\partial q^{p}}\right|_{q=0}
$$

Here, it is assumed that $\hbar$ and $H(\eta)$ are properly chosen in a way that the series solutions converge at $q=1$. Therefore, 


$$
\left.\begin{array}{l}
f(\eta)=f_{0}(\eta)+\sum_{p=1}^{\infty} f_{p}(\eta), \\
h(\eta)=h_{0}(\eta)+\sum_{p=1}^{\infty} h_{p}(\eta), \\
\theta(\eta)=\theta_{0}(\eta)+\sum_{p=1}^{\infty} \theta_{p}(\eta), \\
\phi(\eta)=\phi_{0}(\eta)+\sum_{p=1}^{\infty} \phi_{p}(\eta) .
\end{array}\right\}
$$

\subsection{High-order deformation equations}

In regard to get the higher order deformation equations, the set of solutions is defined by

$$
\left.\begin{array}{r}
f_{n}=\left\{f_{0}(\eta), f_{1}(\eta), f_{2}(\eta), \cdots, f_{n}(\eta)\right\}, \\
h_{n}=\left\{h_{0}(\eta), h_{1}(\eta), h_{2}(\eta), \cdots, h_{n}(\eta)\right\}, \\
\theta_{n}=\left\{\theta_{0}(\eta), \theta_{1}(\eta), \theta_{2}(\eta), \cdots, \theta_{n}(\eta)\right\}, \\
\phi_{n}=\left\{\phi_{0}(\eta), \phi_{1}(\eta), \phi_{2}(\eta), \cdots, \phi_{n}(\eta)\right\} .
\end{array}\right\}
$$

Differentiating the zero-order deformation equations Eq. (20) $p$-times with respect to $q$, for $q=0$, and dividing by $p$ !, yield the $p^{t h}-$ order deformation equations in the following form:

$$
\left.\begin{array}{rl}
\mathcal{L}_{f}\left[f_{p}(\eta)-\mathcal{X}_{p} f_{p-1}(\eta)\right] & =\hbar_{f} H_{f}(\eta) \Re_{p}^{f}\left(f_{p-1}(\eta), h_{p-1}(\eta), \theta_{p-1}(\eta), \phi_{p-1}(\eta)\right), \\
\mathcal{L}_{h}\left[h_{p}(\eta)-\mathcal{X}_{p} h_{p-1}(\eta)\right] & =\hbar_{h} H_{h}(\eta) \Re_{p}^{h}\left(f_{p-1}(\eta), h_{p-1}(\eta), \theta_{p-1}(\eta), \phi_{p-1}(\eta)\right), \\
\mathcal{L}_{\theta}\left[\theta_{p}(\eta)-\mathcal{X}_{p} \theta_{p-1}(\eta)\right] & =\hbar_{\theta} H_{\theta}(\eta) \mathfrak{R}_{p}^{\theta}\left(f_{p-1}(\eta), h_{p-1}(\eta), \theta_{p-1}(\eta), \phi_{p-1}(\eta)\right), \\
\mathcal{L}_{\phi}\left[\phi_{p}(\eta)-\mathcal{X}_{p} \phi_{p-1}(\eta)\right] & =\hbar_{\phi} H_{\phi}(\eta) \Re_{p}^{\phi}\left(f_{p-1}(\eta), h_{p-1}(\eta), \theta_{p-1}(\eta), \phi_{p-1}(\eta)\right)
\end{array}\right\}
$$

with the associated boundary conditions at $\eta=0$ and $\eta \rightarrow \infty$ as follows:

$$
\left.\begin{array}{r}
f_{p}(0)=f_{w} ; f_{p}^{\prime}(0)=s+\alpha f_{p}^{\prime \prime}(0)+\beta f_{p}^{\prime \prime \prime}(0) ; h_{p}(0)=-n f_{p}^{\prime \prime}(0) ; \theta_{p}^{\prime}(0)=-\delta\left(1+\theta_{p}(0)\right) ; \phi_{p}(0)=1, \\
f_{p}^{\prime}(\infty) \rightarrow 0 ; h_{p}(\infty) \rightarrow 0 ; \theta_{p}(\infty) \rightarrow 0 ; \phi_{p}(\infty) \rightarrow 0,
\end{array}\right\}
$$

where

$$
\begin{aligned}
& \mathcal{X}_{p}=\left\{\begin{array}{lll}
0 & \text { for } & p \leq 1 \\
1 & \text { for } & p>1
\end{array}\right. \\
& \mathfrak{R}_{p}^{f}\left(f_{p-1}(\eta), h_{p-1}(\eta), \theta_{p-1}(\eta), \phi_{p-1}(\eta)\right)=(1+K) f_{p-1}^{\prime \prime \prime}(\eta)+\sum_{i=0}^{p-1} f_{i}(\eta) f_{p-1-i}^{\prime \prime}(\eta)-\sum_{i=0}^{p-1} f_{i}^{\prime}(\eta) f_{p-1-i}^{\prime}(\eta)+ \\
& K h_{p-1}^{\prime}(\eta)+\lambda \theta_{p-1}(\eta)+\lambda \Lambda \phi_{p-1}(\eta), \\
& \mathfrak{R}_{p}^{h}\left(f_{p-1}(\eta), h_{p-1}(\eta), \theta_{p-1}(\eta), \phi_{p-1}(\eta)\right)=(1+K / 2) h_{p-1}^{\prime \prime}(\eta)+\sum_{i=0}^{p-1} f_{i}(\eta) h_{p-1-i}^{\prime}(\eta)-\sum_{i=0}^{p-1} f_{i}^{\prime}(\eta) h_{p-1-i}(\eta) \\
& -K\left(2 h_{p-1}(\eta)+f_{p-1}^{\prime \prime}(\eta)\right) \\
& \mathfrak{R}_{p}^{\theta}\left(f_{p-1}(\eta), h_{p-1}(\eta), \theta_{p-1}(\eta), \phi_{p-1}(\eta)\right)=\theta_{p-1}^{\prime \prime}(\eta)+\operatorname{Pr} \sum_{i=0}^{p-1} f_{i}(\eta) \theta_{p-1-i}^{\prime}(\eta) \text { and } \\
& \mathfrak{R}_{p}^{\phi}\left(f_{p-1}(\eta), h_{p-1}(\eta), \theta_{p-1}(\eta), \phi_{p-1}(\eta)\right)=\phi_{p-1}^{\prime \prime}(\eta)+S c \sum_{i=0}^{p-1} f_{i}(\eta) \phi_{p-1-i}^{\prime}(\eta)-S c C_{m} \phi_{p-1}(\eta) .
\end{aligned}
$$

For the sack of simplicity, auxiliary parameters and auxiliary functions are chosen as

$$
\hbar_{f}=\hbar_{g}=\hbar_{h}=\hbar_{\theta}=\hbar \text { and } H_{f}(\eta)=H_{g}(\eta)=H_{h}(\eta)=H_{\theta}(\eta)=1 .
$$


Then, from Eq. (27), the general solutions are

$$
\left.\begin{array}{l}
f_{p}(\eta)=f_{p}^{*}(\eta)+X_{1}+X_{2} e^{\eta}+X_{3} e^{-\eta} \\
h_{p}(\eta)=h_{p}^{*}(\eta)+X_{4}+X_{5} e^{-\eta}, \\
\theta_{p}(\eta)=\theta_{p}^{*}(\eta)+X_{6}+X_{7} e^{-\eta}, \\
\phi_{p}(\eta)=\phi_{p}^{*}(\eta)+X_{8}+X_{9} e^{-\eta},
\end{array}\right\}
$$

where $f_{p}^{*}(\eta), h_{p}^{*}(\eta), \theta_{p}^{*}(\eta)$, and $\phi_{p}^{*}(\eta)$ are particular solutions, and $X_{i}(i=1-9)$ are constants which can be evaluated by using Eq. (28). Furthermore, the current computation is carried out based on the MATHEMATICA software.

\subsection{Region of convergence}

In Homotopy analysis method, the value of the convergence control parameter $(\hbar)$ has an important role on the convergence and the rate of the approximation of the series solution. This value is chosen from the so-called $\hbar$-curves which are normally considered parallel to the horizontal axis. Figs. $2-3$ are computed at $20^{\text {th }}$-order of iterations HAM series solution by using $K=\lambda=\Lambda=S c=C_{m}=f_{w}=n=\delta=0.5, \alpha=1, \beta=-1, \operatorname{Pr}=5$ for stretching $(s=1)$ and shrinking $(s=-1)$ sheets respectively. The admissible ranges of $\hbar$-values for stretching sheet are $-0.3 \leq \hbar_{f}, \hbar_{h}, \hbar_{\phi}<0$ and $-0.25 \leq \hbar_{\theta}<0$. When sheet shrinks, the ranges are considered as $-0.42 \leq \hbar_{f}<0$ and $-0.3 \leq \hbar_{h}, \hbar_{\theta}, \hbar_{\phi}<0$. These values of $\hbar-$ are chosen to validate the HAM series solutions and to analyse impacts of the pertinent parameters on the fluid flow in sections 4 .

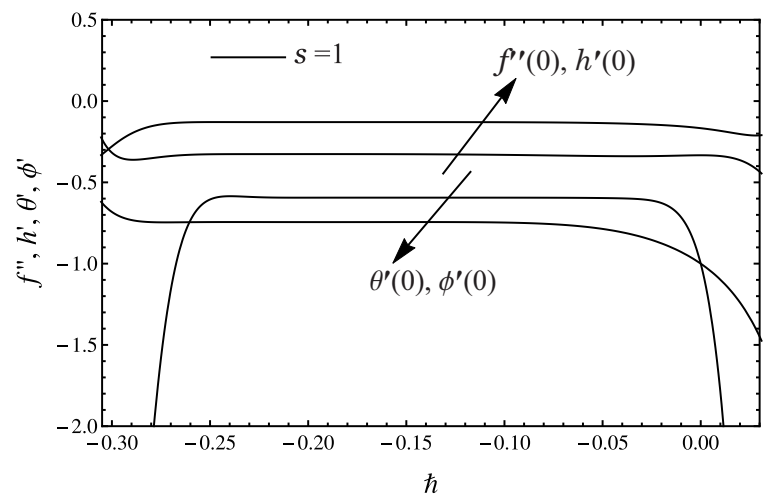

Figure $2 \hbar$-curves

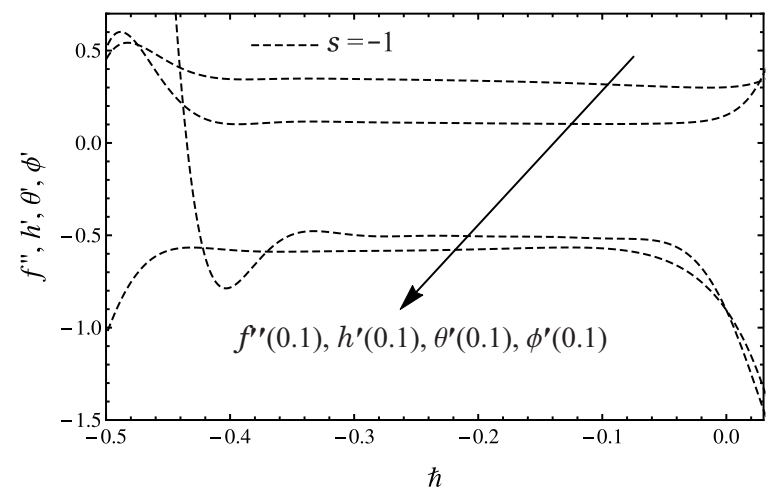

Figure $3 \hbar$-curves

\section{Study Validation}

The validation of the current study is carried out in two special ways. Firstly, the present HAM results are compared with exact analytical results of Fang et al.'s work [23] and numerical results presented by Rosca and Pop [27] for the micropolar fluid flow over a permeable shrinking sheet with the second order slip flow model. It is observed that our results have an excellent agreement with already published results as tabulated in Table 1.

Secondly, the accuracy of the HAM results is also assessed numerically with the help of the three-stage Lobatto IIIA method (please see [27] and references therein). This numerical technique is considered to be very good in the higher order of accuracy for the boundary value problems. For this purpose, the MATLAB generated code bvp4c is used which is based on the three-stage collocation at Lobatto points. Furthermore, solved examples and helping material related to bvp4c can be found in the book of the Shampine et al. [35] and online tutorials by Shampine et al. [36]. For this validation, we choose the maximum value of $\eta$ to be 10 and the same parametric values are used as for Figs. $2-3$ and $\hbar=-0.12,-0.1,-0.1,-0.05$, and $\hbar=-0.402,-0.08,-0.075,-0.07$ are adjusted for velocity component $\left(f^{\prime}\right)$, micro-rotational velocity $(h)$, temperature $(\theta)$ and concentration $(\phi)$ profiles in Fig. 4 and Fig. 5 , respectively. 
Table 1 Current study validation with exact and numerical results

\begin{tabular}{ccccccc}
\hline & & & {$[23]$} & {$[27]$} & Present & \\
\hline$f_{w}$ & $\alpha$ & $\beta$ & $-f^{\prime \prime}(0)$ & $-f^{\prime \prime}(0)$ & $-f^{\prime \prime}(0)$ & $\hbar$ \\
\hline 2.0 & 0.5 & -1 & 0.341213 & 0.3412 & 0.341214 & -0.20629 \\
2.0 & 0.5 & -2 & 0.203824 & 0.2038 & 0.203825 & -0.3074 \\
2.0 & 1.0 & -1 & 0.290548 & 0.2905 & 0.29054 & -0.2068 \\
2.0 & 1.0 & -2 & 0.184657 & 0.1846 & 0.184657 & -0.31136 \\
\hline 3.0 & 0.5 & -1 & 0.262681 & 0.2627 & 0.262681 & -0.209816 \\
3.0 & 0.5 & -2 & 0.147012 & 0.1470 & 0.147012 & -0.273175 \\
3.0 & 1.0 & -1 & 0.232017 & 0.2320 & 0.232014 & -0.2132 \\
3.0 & 1.0 & -2 & 0.136905 & 0.1369 & 0.136905 & -0.27912 \\
\hline
\end{tabular}

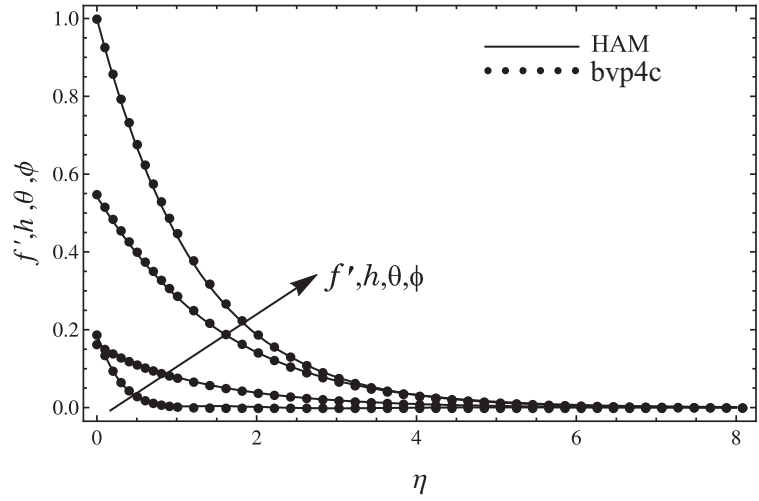

Figure 4 comparison with bvp4c for

$$
s=1
$$

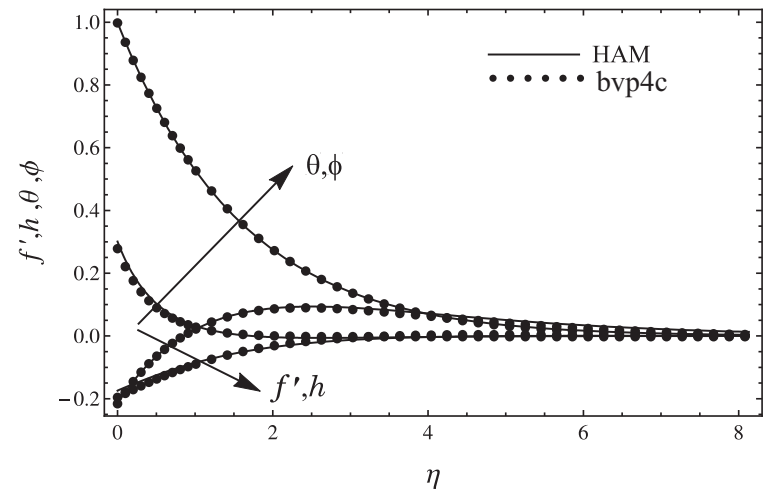

Figure 5 comparison with bvp4c for

$$
s=-1
$$

\section{Results and Discussion}

A mathematical model of the heat and mass transfer micropolar fluid flow over a stretching/shrinking sheet with slip flow model has been formulated, solved and validated in the preceding sections. Now, in this section, the asymptomatic analysis is carried out from the obtained results. The influences of the governing parameters such as material parameter $(K)$, bouncy parameter $(\lambda)$, concentration to thermal bouncy ratio parameter $(\Lambda)$, Prandtl number $(P r)$, Schmidt number $(S c)$, chemical reaction parameter $\left(C_{m}\right)$, suction/injection parameter $\left(f_{w}\right)$, first and second order slip flow parameters $(\alpha, \beta)$, and the Newtonian heating parameters $(\delta)$ along with the physical quantities, i.e., local skin friction coefficient, local wall couple stress, local Nusselt number and the local Sherwood number are reported with the help of graphical and tabular illustrations. It is to be noted that all of the presented results are computed at fixed values of the pertaining parameters which are used to calculate the $\hbar$-curves otherwise stated with description.

Figs. 6-7 depict the influence of the material parameter on the velocity component and the microrotation velocity with the increasing values of the $\eta$ for $\hbar=-0.1,-0.01$, respectively. It is observed that when the concentration of micro-particles increases, the corresponding velocities increase and decrease for $s=1$ and $s=-1$, respectively. In Fig. 6 as the material parameter increases from 0 to 2 , it is observed that the boundary layer thickness increases the 0.06 unit for the stretching case and it decreases 0.23 unit for the shrinking case at $\eta=0$. However, there is a small change in Fig. 7 as the similarity variable $\eta$ moves away from the stretching/shrinking permeable sheet. All the physical quantities increase with the increasing values of the $K$ as tabulated in Table 2, while a reverse pattern is observed in Table 3.

The impacts of the bouncy $(\lambda)$ and the concentration to thermal bouncy ratio $(\Lambda)$ parameters are illustrated in Figs. 8-9 at $\hbar=-0.01$. When the sheet stretches or shrinks, the increase in the bouncy parameter causes to exert a force to raise the velocity component of the micropolar fluid flow as shown in Fig. 8. Moreover, it is also noted that the thickness of the boundary layer in the shrinking case is higher than the stretching case. While concentration to thermal bouncy ratio parameter also depicts the same kind of effect on the velocity component 


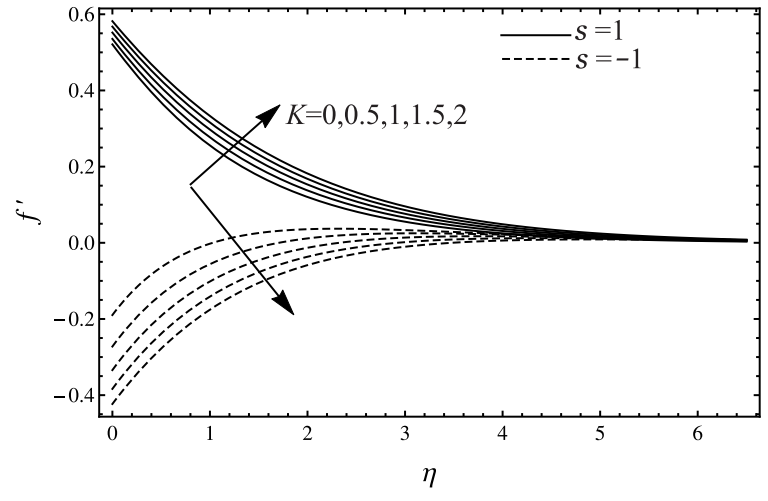

Figure 6 Impact of $K$ on $f^{\prime}$

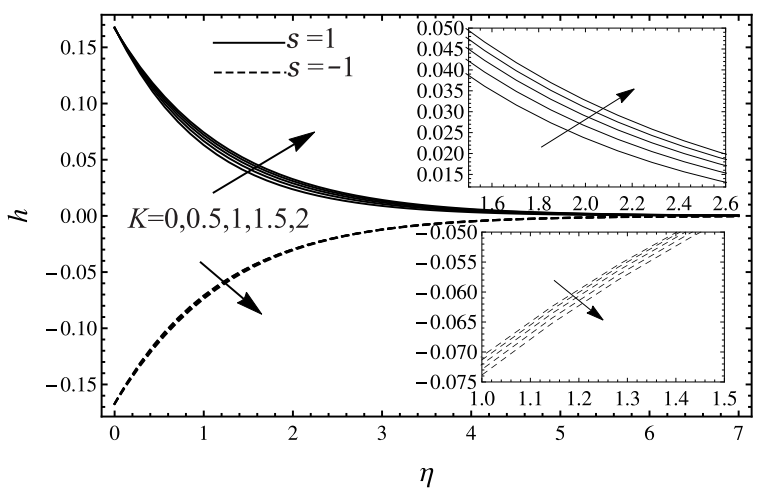

Figure 7 Impact of $K$ on $h$

in Fig. 9. Table 4 shows that an increase in the bouncy parameter $(\lambda)$ for stretching sheet leads an increase in all of the physical quantities. On the other hand in Table 5 for shrinking sheet, it reduces the local skin friction and local wall couple stress and raises the local Nusselt and Sherwood number. When sheet stretches or shrinks, increasing values of concentration to thermal bouncy ratio $(\Lambda)$ causes to decrease the local skin friction and the local wall couple stress while it raises the heat and mass transfer rate as shown in the Tables 2-3.

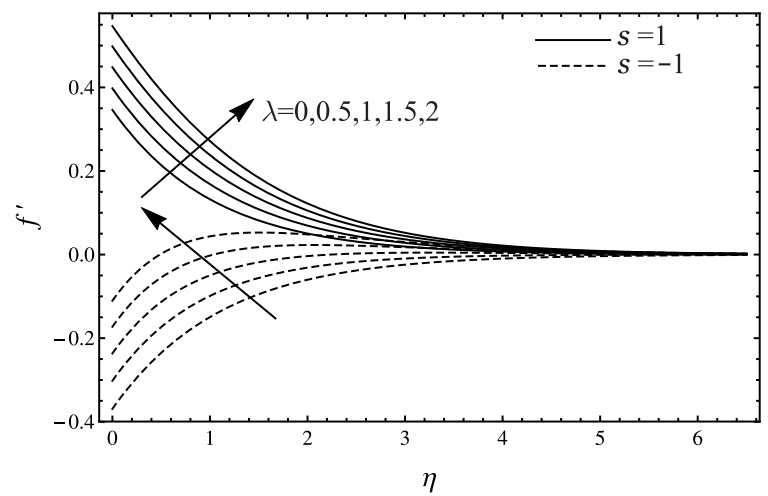

Figure 8 Impact of $\lambda$ on $f^{\prime}$

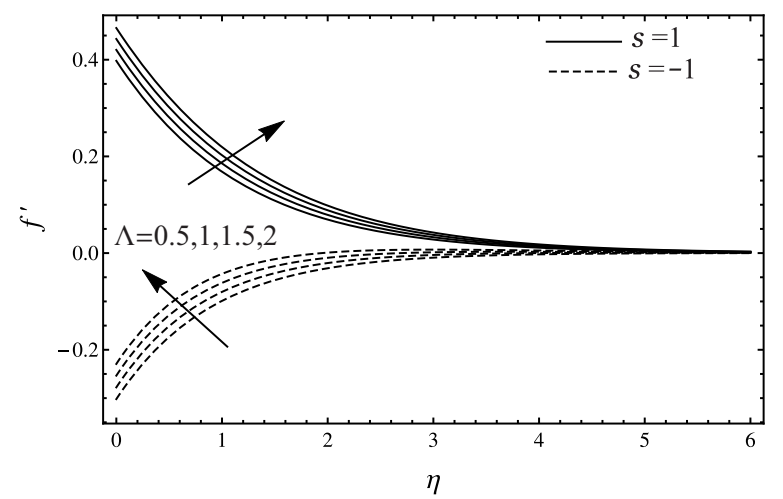

Figure 9 Impact of $\Lambda$ on $f^{\prime}$

Various values of $\operatorname{Pr}$ are chosen to investigate it's influence on the thermal boundary layer thickness. The results as shown in Fig. 10 using $\hbar=-0.01,-0.01,-0.01,-0.001,-0.001,-0.001,-0.0006,-0.0004,-0.0001$ are used for stretching case and $\hbar=-0.009,-0.007-0.005,-0.0001,-0.001,-0.001,-0.0006,-0.0004,-0.0001$ corresponding to the increasing values of the $P r$ indicate that increasing values of the $P r$, an increase in the momentum diffusivity, causes to reduce the fluid temperature for $s=1,-1$. It is also observed that stretching sheet yields a wider thickness of the thermal boundary as compared to the shrinking of the sheet. All the physical quantities increase with the increasing values of the $\mathrm{Pr}$ in Tables 4-5, expect for shrinking case, only local wall couple stress decreases.

Fig. 11 displays the impact of the Schmidt number $(S c)$ on the concentration of species at $\hbar=-0.05,-0.04$ for stretching and shrinking the sheet, respectively. It is well known that dimensionless parameter $S c$ is a ratio of viscous diffusion rate to molecular diffusion rate. A decrease in the mass diffusion rate, i.e., an increase in the $S c$ leads to a decrease in the concentration. The plot also shows that the shrinking sheet reduces more concentration of the micropolar fluid flow in comparison with the stretching sheet. However, an increase in the Schmidt number yields a decrease in the heat transfer rate and an increase in all other physical quantities as shown in the Tables 2-3.

Figs. 12-13 illustrate the effects of first and second order slip flow parameters on the velocity component at $\hbar=-0.01$. When sheet stretches, the velocity component decreases and it increases with the shrinking of the sheet. All physical quantities decrease when the sheet stretches and increase with the shrinking of the sheet in the presence of the higher values of the first and second order slip flow parameters in Tables 4-5. Fig. 14 depicts the influence of the suction and injection parameter $\left(f_{w}\right)$ on the velocity component at $\hbar=-0.01$ and -0.008 for stretching and shrinking of the sheet, respectively. In both cases, it shows that an increase in the suction parameter causes a decrease in the velocity component and an increase in the injection parameter raises the velocity component 


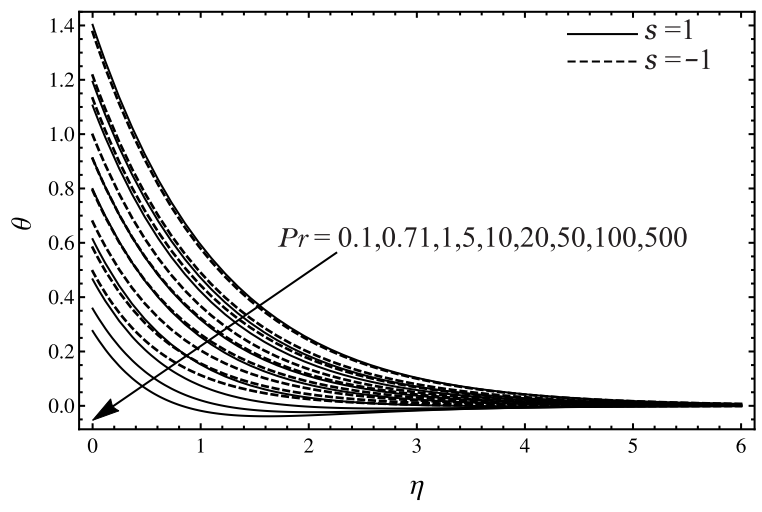

Figure 10 Impact of $\operatorname{Pr}$ on $\theta$

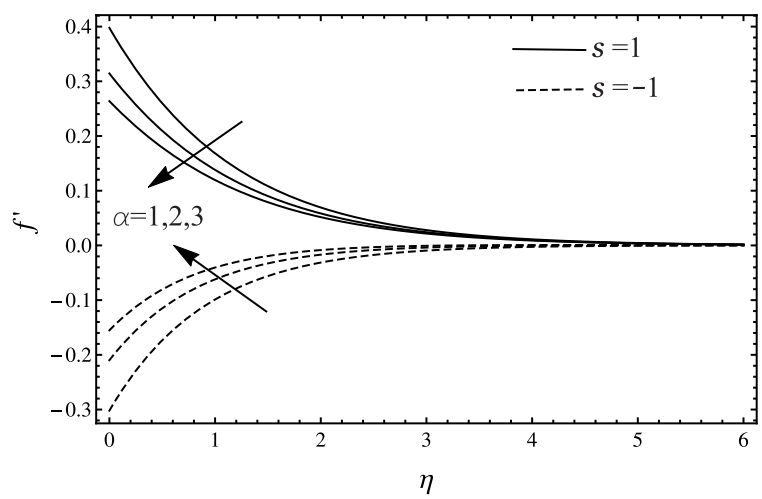

Figure 12 Impact of $\alpha$ on $f^{\prime}$

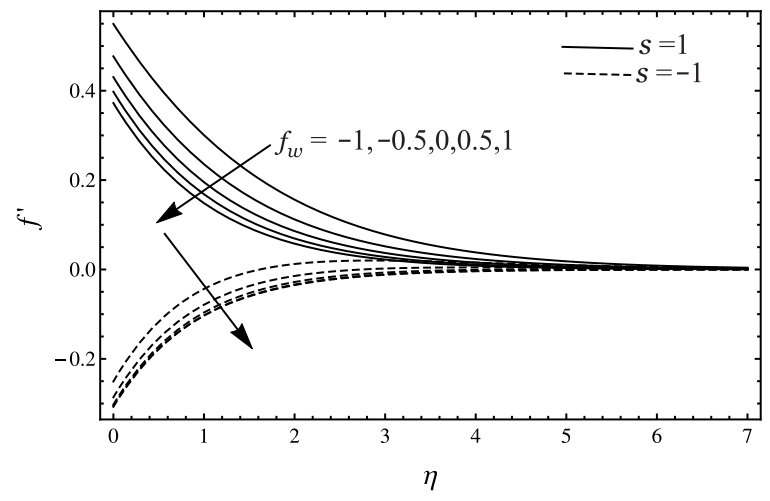

Figure 14 Impact of $f_{w}$ on $f^{\prime}$

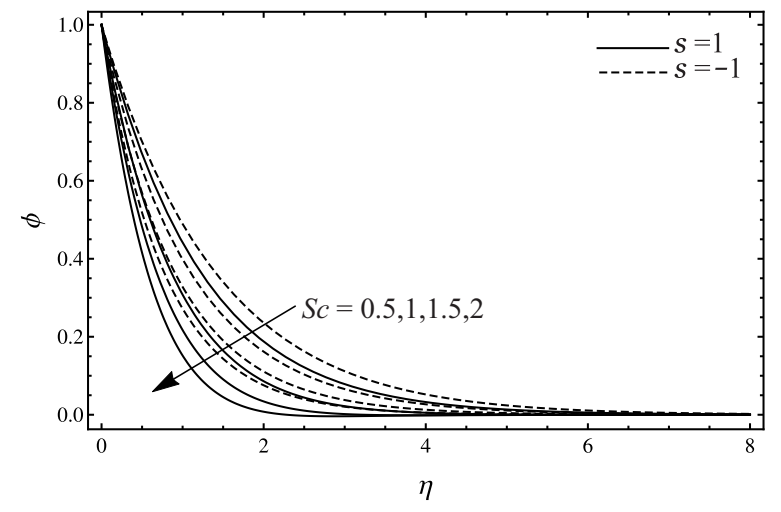

Figure 11 Impact of $S c$ on $\phi$

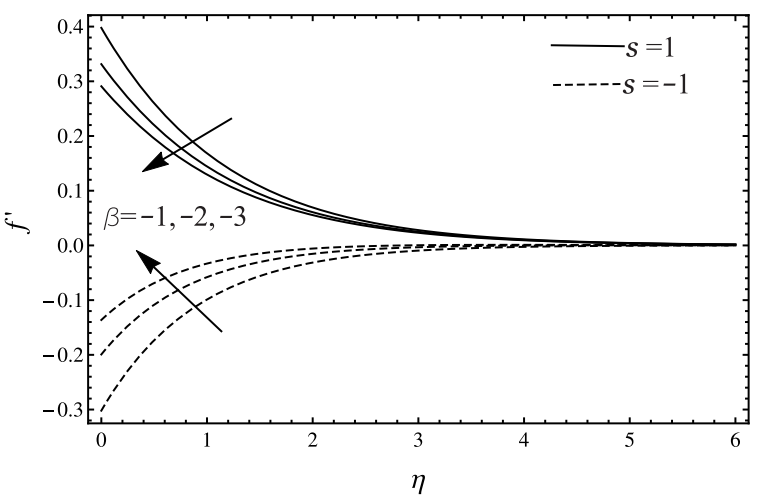

Figure 13 Impact of $\beta$ on $f^{\prime}$

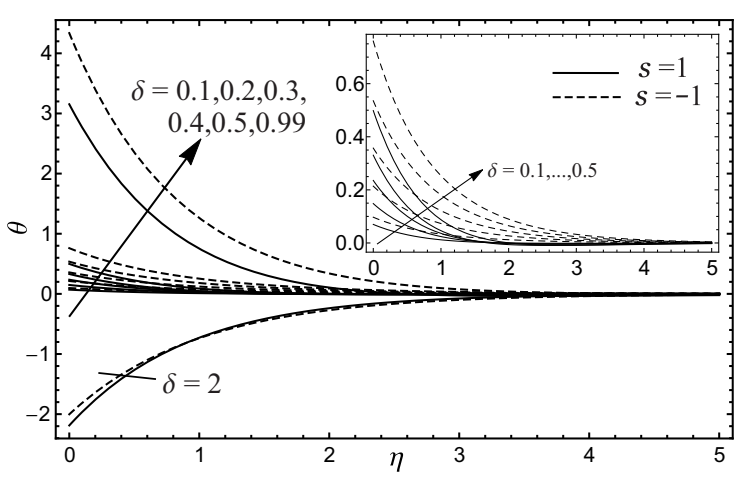

Figure 15 Impact of $\delta$ on $\theta$ 


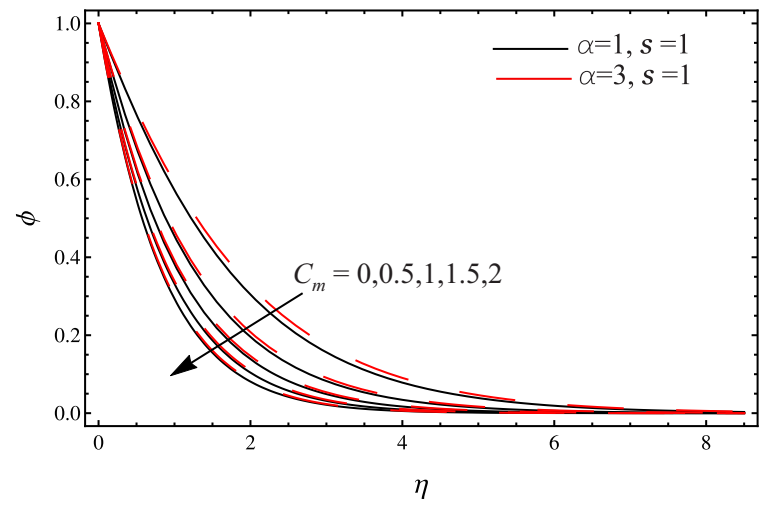

Figure 16 Impact of $C_{m}$ on $\phi$

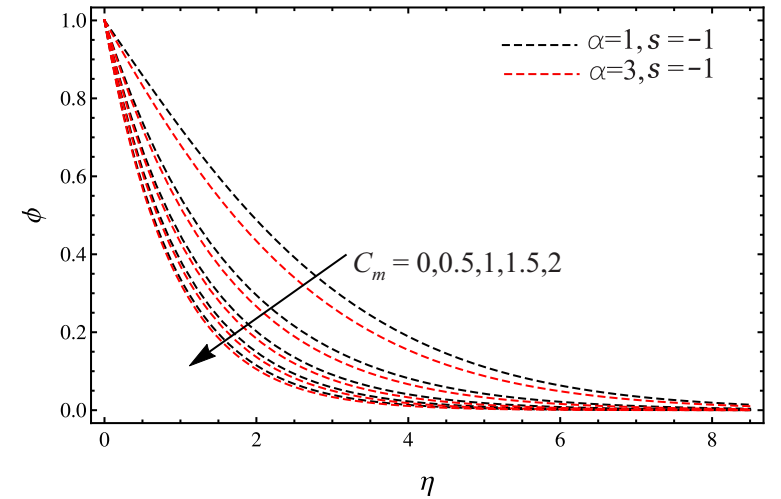

Figure 17 Impact of $C_{m}$ on $\phi$

with the increasing values of the similarity variable $\eta$. Table 2 displays a decrease in the mass transfer rate and an increase in the local skin friction, wall couple stress and heat transfer rate with the increasing values of the suction parameter and on the other hand in Table 3, local skin friction and heat transfer rate increase, and local wall couple stress and mass transfer rate decrease. An opposite behaviour is noted with the increasing values of the injection parameter. For different values of the Newtonian heating parameter $(\delta)$, the temperature profile is plotted in Fig. 15. It is obvious that increasing values of the Newtonian heating parameter causes to raise the fluid temperature and the thermal boundary layer thickness thus increases. It is noted that the shrinking sheet produces a higher thermal boundary layer thickness as compared to the stretching sheet, and with a 31.6 percentage difference at $\eta=0$ for $\delta=0.99$. On the other hand, for $\delta=2$ the temperature of the micropolar fluid flow starts decreasing. The auxiliary parameter $(\hbar)$ for stretching sheet is chosen to be $-0.008,-0.00055$ and -0.002 for $\delta=0.5,0.99$ and 2 , respectively. For shrinking sheet $\hbar=-0.0008$ and -0.0001 for $\delta=0.99$ and 2, respectively. Moreover, rest of the curves generated from other values of the $\delta$ are displayed at $\hbar=-0.01$. When sheet stretches, higher values of the Newtonian heating parameter produce an increase in all of the physical quantities as tabulated in Table 4 and when sheet shrinks, local skin friction and wall couple stress decrease, and heat and mass transfer rate increase as depicted in Table 5.

Figs. 16-23 are plotted to analyse the impact of the first order chemical reaction parameter on the concentration profile of the micropolar fluid flow at $\hbar=-0.1$ with the asymptotically increasing curves. All these results show that the concentration profile decreases by increasing the chemical reaction parameter. More importantly, in contrast to the stretching sheet, when sheet shrinks the thickness of the concentration boundary layer at the lower values of $\alpha, \beta, \Lambda$ and $\delta$ is wider than that at their higher values. For stretching sheet, there is a small difference in the concentration boundary layer thicknesses for different values values of $\alpha, \beta, \Lambda$ and $\delta$. An increase in the chemical reaction parameter causes to raise the local skin friction, wall couple stress and mass transfer rate, and reduce the heat transfer rate as shown in Tables 2-3. It is interesting to observe that the mass transfer rate for the stretched sheet was $281.35 \%$ more than that of the shrunk sheet.

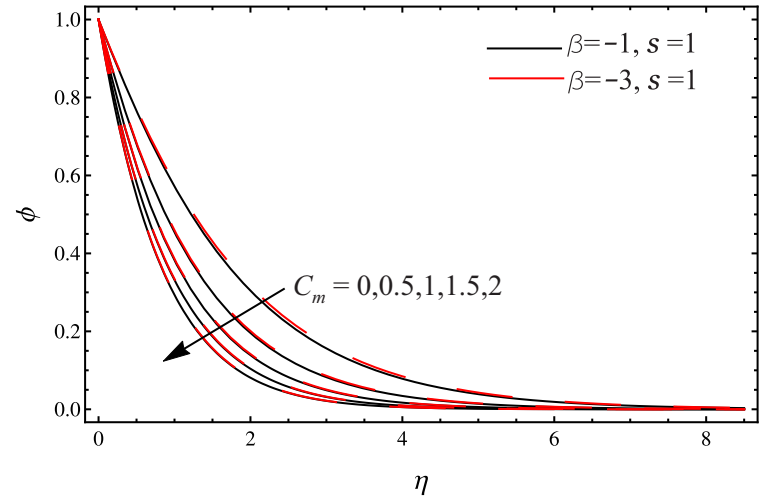

Figure 18 Impact of $C_{m}$ on $\phi$

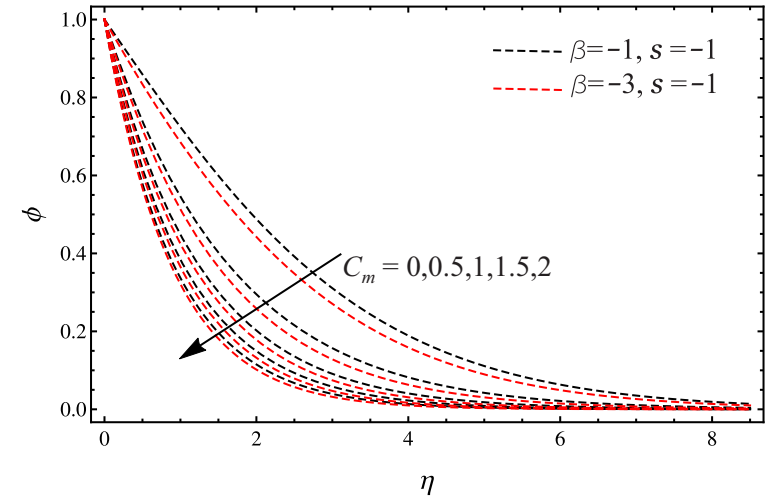

Figure 19 Impact of $C_{m}$ on $\phi$ 


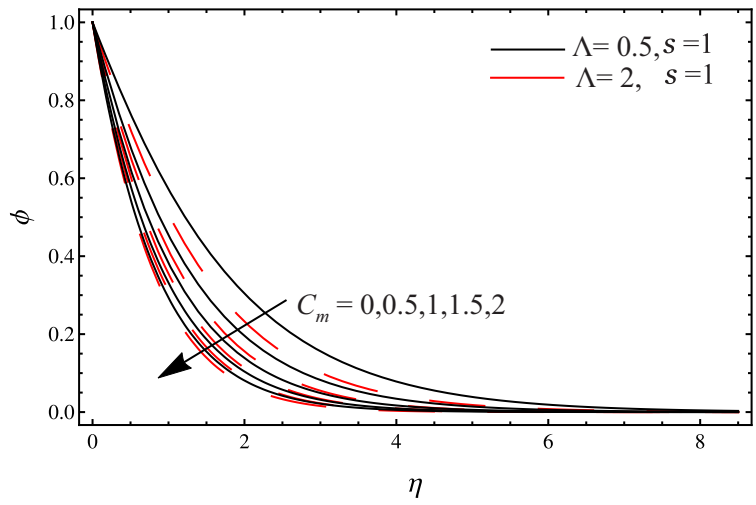

Figure 20 Impact of $C_{m}$ on $\phi$

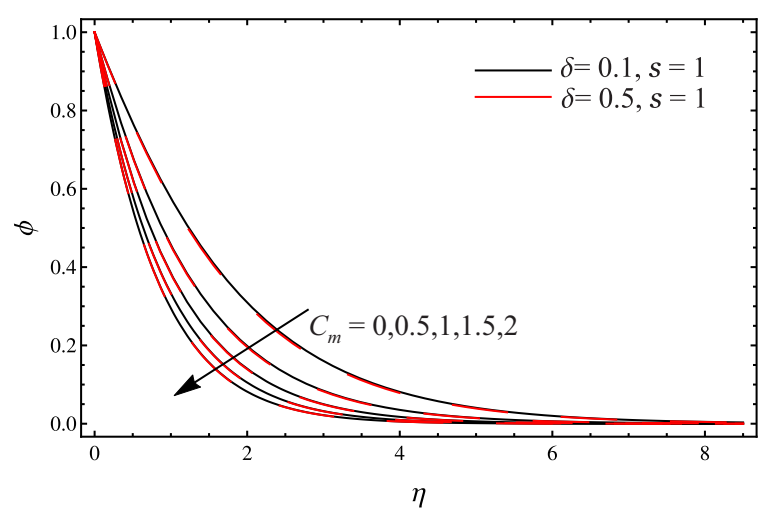

Figure 22 Impact of $C_{m}$ on $\phi$

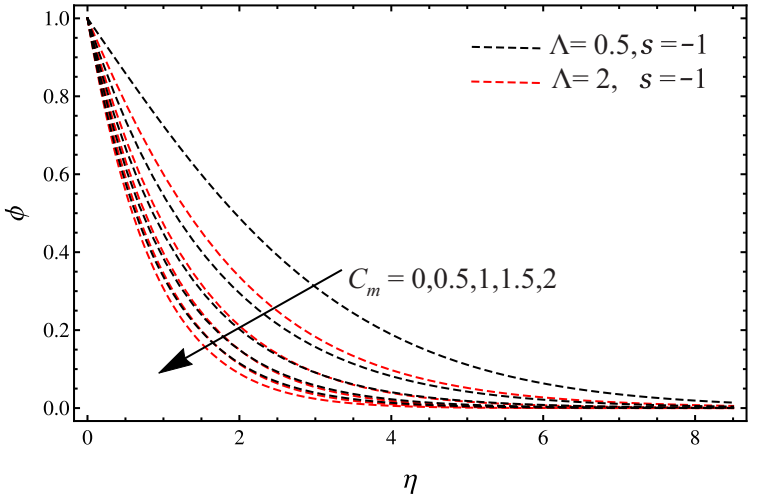

Figure 21 Impact of $C_{m}$ on $\phi$

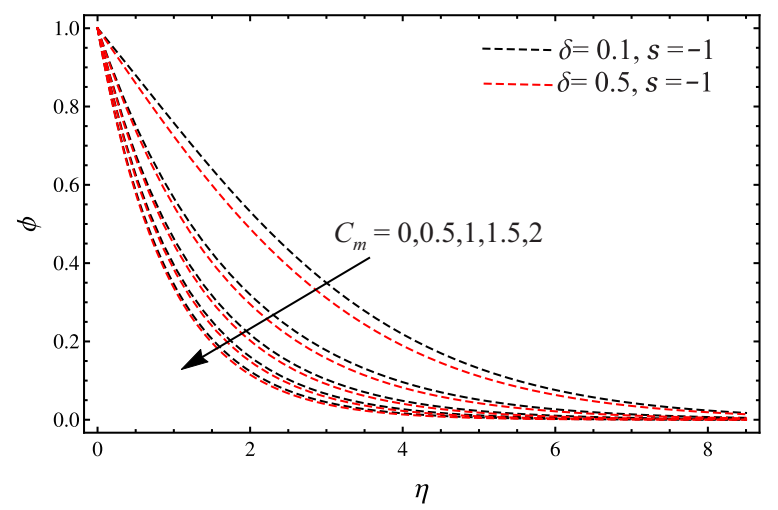

Figure 23 Impact of $C_{m}$ on $\phi$

Table 2 Numerical illustrations of physical quantities for stretching sheet $(s=1)$.

\begin{tabular}{|c|c|c|c|c|c|c|c|c|c|c|}
\hline$K \Lambda$ & $S c C_{m}$ & $f_{w}$ & $C_{f_{x}} R_{e_{x}}^{\overline{\overline{2}}}$ & $\hbar$ & $M_{x} R e_{x}$ & $\hbar$ & $N u_{x} R e_{x}^{-\frac{1}{2}}$ & $\hbar$ & $S h_{x} R e_{x}^{-\frac{1}{2}}$ & $\hbar$ \\
\hline 0.01 & 0.50 .5 & 0.5 & 0.343718 & -0.1 & 0.164237 & -0.01 & 1.65823 & -0.01 & 0.741868 & -0.1 \\
\hline 0.5 & & & 0.413585 & -0.1 & 0.19393 & -0.01 & 1.66467 & -0.01 & 0.747196 & -0.1 \\
\hline 1.0 & & & 0.48229 & -0.1 & 0.221043 & -0.01 & 1.67119 & -0.01 & 0.752522 & -0.1 \\
\hline 1.5 & & & 0.548835 & -0.1 & 0.246008 & -0.01 & 1.67776 & -0.01 & 0.757547 & -0.1 \\
\hline 2.0 & & & 0.613072 & -0.1 & 0.269128 & -0.01 & 1.68437 & -0.01 & 0.762226 & -0.1 \\
\hline 0.50 .5 & & & 0.418933 & -0.01 & 0.19393 & -0.01 & 1.66467 & -0.01 & 0.928328 & -0.01 \\
\hline 1.0 & & & 0.418389 & -0.01 & 0.193132 & -0.01 & 1.68613 & -0.01 & 0.929113 & -0.01 \\
\hline 1.5 & & & 0.417861 & -0.01 & 0.192342 & -0.01 & 1.70818 & -0.01 & 0.929897 & -0.01 \\
\hline 2.0 & & & 0.417348 & -0.01 & 0.191561 & -0.01 & 1.73086 & -0.01 & 0.930681 & -0.01 \\
\hline 0.5 & 0.5 & & 0.413585 & -0.1 & 0.162428 & -0.1 & 1.66467 & -0.01 & 0.747196 & -0.1 \\
\hline & 1.0 & & 0.431967 & -0.1 & 0.169961 & -0.1 & 1.66426 & -0.01 & 1.14879 & -0.1 \\
\hline & 1.5 & & 0.443108 & -0.1 & 0.174908 & -0.1 & 1.66387 & -0.01 & 1.48886 & -0.1 \\
\hline & 2.0 & & 0.450715 & -0.1 & 0.178513 & -0.1 & 1.66351 & -0.01 & 1.80004 & -0.1 \\
\hline & 0.50 .0 & & 0.401407 & -0.1 & 0.157571 & -0.1 & 1.66511 & -0.01 & 0.121577 & -0.1 \\
\hline & 0.5 & & 0.413585 & -0.1 & 0.162428 & -0.1 & 1.66467 & -0.01 & 0.747196 & -0.1 \\
\hline & 1.0 & & 0.4 & -0.1 & 703 & -0.1 & 1.66426 & -0.01 & 1.14879 & -0.1 \\
\hline & 1.5 & & 0.427491 & -0.1 & 3131 & -0.1 & 1.66387 & -0.01 & 1.48886 & -0.1 \\
\hline & 2.0 & & 0.43199 & -0.1 & 0.17005 & -0.1 & 1.66351 & -0.01 & 1.80004 & -0.1 \\
\hline & 0.5 & 1.0 & 0.42098 & -0.01 & 0.21229 & -0.01 & 1.74435 & -0.005 & 0.79984 & -0.01 \\
\hline & & 0.5 & 0.418933 & -0.0 & 0.19393 & -0.01 & 1.28438 & -0.005 & 0.84223 & -0.01 \\
\hline & & 0.0 & 0.414743 & -0.0 & 0.17462 & -0.01 & 0.99266 & -0.005 & 0.88512 & -0.01 \\
\hline & & & 6697 & -0.0 & 0.153562 & -0.01 & 0.80963 & -0.005 & 0.92833 & -0.01 \\
\hline & & -1 & 0.391548 & -0.01 & 0.129237 & -0.01 & 0.69529 & -0.005 & 0.97176 & -0.01 \\
\hline
\end{tabular}


Table 3 Numerical illustrations of physical quantities for shrinking sheet $(s=-1)$.

\begin{tabular}{|c|c|c|c|c|c|c|c|c|c|c|}
\hline$K \quad \Lambda$ & $S c$ & $C_{m} f_{w}$ & $C_{f_{x}} R e_{x}$ & $\hbar$ & $M_{x} R e_{x}$ & $\hbar$ & $N u_{x} R^{-\frac{1}{2}} e_{x}$ & $\hbar$ & $S h_{x} R e_{x}^{-\frac{1}{2}}$ & $\hbar$ \\
\hline 0.01 & 0.5 & 0.50 .5 & -0.345545 & -0.1 & -0.142159 & -0.01 & 1.16213 & -0.01 & 0.62335 & -0.1 \\
\hline 0.5 & & & -0.441393 & -0.1 & -0.174183 & -0.01 & 1.15765 & -0.01 & 0.607856 & -0.1 \\
\hline 1.0 & & & -0.529613 & -0.1 & -0.204601 & -0.01 & 1.15325 & -0.01 & & -0.1 \\
\hline 1.5 & & & -0.611233 & -0.1 & -0.233402 & -0.01 & 1.14894 & -0.01 & 0.582713 & -0.1 \\
\hline 2.0 & & & -0.687326 & -0.1 & -0.26061 & -0.01 & 1.14471 & -0.01 & 0.572009 & -0.1 \\
\hline 0.50 .5 & & & -0.41762 & -0.01 & -0.136017 & -0.05 & 1.15765 & -0.01 & 97204 & -0.01 \\
\hline 1.0 & & & -0.418684 & -0.01 & -0.166335 & -0.05 & 1.17042 & -0.01 & 0.898047 & -0.01 \\
\hline 1.5 & & & -0.419731 & -0.01 & -0.195537 & -0.05 & 1.18359 & -0.01 & 98889 & -0.01 \\
\hline 2.0 & & & -0.42076 & -0.01 & -0.223616 & -0.05 & 1.19716 & -0.01 & 0.89973 & -0.01 \\
\hline 0.5 & 0.5 & & -0.441393 & -0.1 & -0.129694 & -0.1 & 1.15765 & -0.01 & 0.607856 & -0.1 \\
\hline & 1.0 & & -0.423962 & -0.1 & -0.120326 & -0.1 & 1.15751 & -0.01 & 32725 & -0.1 \\
\hline & 1.5 & & -0.412188 & -0.1 & -0.113768 & -0.1 & 1.15738 & $\begin{array}{l}-0.01 \\
-0.01\end{array}$ & 120634 & -0.1 \\
\hline & 2.0 & & -0.403465 & -0.1 & -0.108751 & -0.1 & 1.15726 & -0.01 & 1.45798 & -0.1 \\
\hline & 0.5( & 0.0 & -0.460383 & -0.1 & -0.139606 & -0.1 & 1.15779 & -0.01 & 0.121577 & -0.1 \\
\hline & & 0.5 & -0.441393 & -0.1 & -0.129694 & -0.1 & 1.15765 & -0.01 & 0.607856 & -0.1 \\
\hline & & 1.0 & -0.429429 & -0.1 & -0.123341 & -0.1 & 1.15751 & -0.01 & 0.932725 & -0.1 \\
\hline & & 1.5 & -0.421077 & -0.1 & -0.118812 & -0.1 & 1.15738 & -0.01 & 1.20634 & -0.1 \\
\hline & & 2.0 & -0.414796 & -0.1 & -0.115336 & -0.1 & 1.15726 & -0.01 & 1.45798 & -0.1 \\
\hline & & 0.51 .0 & -0.414304 & -0.01 & -0.208091 & -0.00 & 1.43749 & -0.005 & 0.76556 & -0.01 \\
\hline & & 0.5 & -0.41762 & -0.01 & -0.207883 & -0.0001 & 1.07259 & -0.005 & 0.80881 & -0.01 \\
\hline & & 0. & -0.424091 & -0.0 & -0.207675 & -0.0001 & 0.851961 & -0.005 & 0.85279 & -0.01 \\
\hline & & $-\dot{0}$ & -0.436899 & -0.0 & -0.207468 & -0.0001 & 0.718105 & -0.005 & 0.89720 & -0.01 \\
\hline & & -1 . & -0.462025 & -0.01 & -0.20726 & -0.0001 & 0.636378 & -0.005 & 0.94189 & -0.01 \\
\hline
\end{tabular}

Table 4 Numerical illustrations of physical quantities for stretching sheet $(s=1)$.

\begin{tabular}{|c|c|c|c|c|c|c|c|c|c|c|c|}
\hline$\delta$ & $\lambda$ & $\operatorname{Pr}$ & $\alpha \beta$ & $C_{f_{x}} R^{\frac{1}{2}} e_{x}$ & $\hbar$ & $M_{x} R e_{x}$ & $\hbar$ & $N u_{x} R e_{x}^{-\frac{1}{2}}$ & $\hbar$ & $S h_{x} R e_{x}^{-\frac{1}{2}}$ & $\hbar$ \\
\hline 0.1 & 1 & 5 & $1-1$ & 0.398412 & -0.1 & 0.15604 & -0.1 & 1.53027 & -0.01 & 0.743175 & -0.1 \\
\hline 0.2 & & & & 0.401825 & -0.1 & .15745 & -0.1 & 1.5490 & -0.01 & 0.744116 & -0.1 \\
\hline 0.3 & & & & 0.405477 & -0.1 & 0.15898 & -0.1 & 1.57427 & -0.01 & 0.745098 & -0.1 \\
\hline 0.4 & & & & 0.409389 & -0.1 & 0.16063 & -0.1 & 1.61012 & -0.01 & 0.746124 & -0.1 \\
\hline 0.5 & & & & 0.413585 & -0.1 & . 16243 & -0.1 & 1.66467 & -0.01 & 0.747196 & -0.1 \\
\hline 05 & 0.0 & & & 0.416618 & -0.01 & .19294 & -0.011 & 1.61716 & -0.01 & 0.92643 & -0.01 \\
\hline & 0.5 & & & 0.41893 & -0.01 & 0.19393 & -0.01 & 1.66467 & -0.01 & 0.92833 & -0.01 \\
\hline & 1.0 & & & & -0.01 & & -0.01 & 1431 & -0.01 & 0.93021 & -0.01 \\
\hline & 1.5 & & & 439 & -0.01 & 12 & -0.01 & 1.76615 & -0.01 & 0.93206 & -0.01 \\
\hline & 2.0 & & & 0.42752 & -0.01 & 44 & -0.01 & 1.82027 & -0.01 & 0.93389 & -0.01 \\
\hline & 0.5 & 0.1 & & 0.41351 & -0.01 & 787 & -0.01 & 0.856171 & -0.01 & 0.929012 & -0.01 \\
\hline & & 0.71 & & 454 & -0.01 & 137 & -0.01 & 6314 & -0.01 & 0.991797 & -0.001 \\
\hline & & & & 0.41498 & -0.01 & 0. & -0.01 & 8121 & -0.01 & 0.998339 & -0.0002 \\
\hline & & 5 & & 670 & -0.001 & 0.206629 & -0.001 & 1.04865 & -0.001 & 0.999168 & -0.0001 \\
\hline & & 10 & & 677 & -0.001 & 0.206661 & -0.001 & 1.12604 & -0.001 & 0.999917 & -0.00001 \\
\hline & & 20 & & 687 & -0.001 & 0.20 & -0.001 & 1.31341 & -0.001 & 0.999933 & -0.000 \\
\hline & & 50 & & 707 & -0.001 & 0.2 & -0.0006 & 1.5719 & -0.0006 & 0.99995 & -0.0000 \\
\hline & & 100 & & 0.41780 & -0.01 & 0.2 & -0.0004 & 1.89454 & -0.0004 & 0.999967 & -0.000004 \\
\hline & & 500 & & 0.42185 & -0.0031 & 181 & -0.0001 & 47 & -0.0001 & 0.999992 & -0.000001 \\
\hline & & 5 & & 0.41359 & -0.1 & 0.1 & -0.1 & 1.6 & -0.1 & 0.74720 & -0.1 \\
\hline & & & & 0.30744 & -0.1 & 0.1 & -0.1 & & -0 . & & -0.1 \\
\hline & & & & & -0.1 & & -0.1 & & -0.1 & 0.7 & -0.1 \\
\hline & & & & 0.41893 & -0.01 & 0.19393 & -0.01 & 1.6 & -0.01 & 0.9 & -0.01 \\
\hline & & & & & -0.01 & & & & -0.01 & & -0.01 \\
\hline & & & & & -0.01 & 16 & -0.01 & 1.57586 & -0.01 & 0.92303 & -0.01 \\
\hline
\end{tabular}


Table 5 Numerical illustrations of physical quantities for shrinking sheet $(s=-1)$.

\begin{tabular}{|c|c|c|c|c|c|c|c|c|c|c|c|}
\hline$\delta$ & $\lambda$ & $\operatorname{Pr}$ & $\alpha \beta$ & $C_{f_{x}} R e_{x}^{\frac{1}{2}}$ & $\hbar$ & $M_{x} R e_{x}$ & $\hbar$ & $N u_{x} R e_{x}^{-\frac{1}{2}}$ & $\hbar$ & $S h_{x} R_{e_{x}^{-\frac{1}{2}}}$ & $\hbar$ \\
\hline 0 . & 1 & 5 & $1-1$ & -0.42592 & -0.05 & -0.08983 & -0.1 & 1.13073 & -0.01 & 0.577924 & -0.1 \\
\hline 0 & & & & -0.42709 & -0.05 & 106 & -0.1 & 3459 & -0.01 & 5609 & -0.1 \\
\hline $4 ?$ & & & & -0.42795 & -0.05 & -0.11169 & -0.1 & 1.13972 & -0.01 & 0.59338 & -0.1 \\
\hline & & & & -0.42817 & -0.05 & -0.12124 & -0.1 & & -0.01 & 0.60089 & -0.1 \\
\hline 0.5 & & & & -0.42990 & -0.06 & -0.12969 & -0.1 & 1.15765 & -0.01 & 0.60786 & -0.1 \\
\hline 0.5 & 0.0 & & & -0.41606 & -0.01 & -0.06392 & -0.05 & 1.12377 & -0.01 & 0.89483 & -0.01 \\
\hline & 0.5 & & & -0.41762 & -0.01 & 02 & 5 & & 1 & 720 & -0.01 \\
\hline & 1.0 & & & 868 & -0.01 & 076 & -0.05 & 1.19396 & -0.01 & 9954 & -0.01 \\
\hline & 1.5 & & & 926 & -0.01 & 136 & -0.05 & 1.23291 & -0.01 & 0.90185 & -0.01 \\
\hline & 2.0 & & & -0.41936 & -0.01 & & -0.05 & 1.27475 & -0.01 & 0.90412 & -0.01 \\
\hline & 0.5 & 0.1 & & -0.420614 & -0.01 & 59 & 0.009 & 0.851493 & -0.01 & 0.897603 & -0.01 \\
\hline & & 0.71 & & 0167 & -0.01 & 156 & & 9408 & -0.01 & 482 & -0.001 \\
\hline & & 7 & & 9963 & -0.01 & 859 & -0. & 3503 & -0.01 & 673 & -0.0002 \\
\hline & & 5 & & -0 . & -0.001 & -0.2 & -0. & 356 & -0.001 & 35 & -0.0001 \\
\hline & & 10 & & 52 & -0.001 & -0 & -0 . & 15 & -0.001 & 883 & -0.00001 \\
\hline & & 20 & & -0.4 & -0.001 & -0. & -0.0 & 1.12918 & -0.001 & 907 & -0.00( \\
\hline & & 50 & & & -0.001 & & & & -0.0006 & & -0.00 \\
\hline & & 100 & & & -0.001 & -0.2 & -0 . & 1.0 & -0.0004 & 9953 & -0.000 \\
\hline & & 500 & & -0.416248 & -0.001 & 872 & -0.0001 & 1.50871 & -0.0001 & 0.999988 & -0.000001 \\
\hline & & 5 & 1 & & -0.1 & & -0 & & -0.1 & 856 & -0.1 \\
\hline & & & & & -0 & 33 & -0 & 1.2 & -0 & 2404 & -0 \\
\hline & & & $J$ & -0.26341 & -0.1 & 825 & -0 & 1.25154 & -0.1 & 6396 & -0.1 \\
\hline & & & & & -0.01 & & & & -0.01 & 0.897204 & -0.01 \\
\hline & & & & & & -0 . & & 1.21981 & -0.01 & 0.90155 & -0.01 \\
\hline & & & & -0.22759 & -0.01 & -0.09867 & -0.01 & 1.25955 & -0.01 & 0.90417 & -0.01 \\
\hline
\end{tabular}

\section{Conclusion}

In this paper, we reported the asymptomatic analysis of the chemical reaction and the Newtonian heating parameters on an incompressible mixed convective micropolar fluid flow. A second-order slip flow model was considered in the presence of the dilute concentration of microparticles. Semi-analytical results obtained from the Homotopy Analysis Method were, in all cases, found to give an excellent comparison with the already published analytical and numerical results. It was found that when sheet stretched, the thickness of the concentration boundary layer became shorter with a small change in the chemical reaction parameter. On the other hand with the shrunk sheet, an increase in the values of the chemical reaction parameter produced a wider concentration boundary layer thickness at the lower values of the pertaining parameters. It was interesting to observe that the mass transfer rate for the stretched sheet was very high in comparison to the shrunk sheet. As expected, first and second order slip flow parameters reduced the velocity component for the stretched sheet, and an opposite trend was noted with the shrunk sheet. Furthermore, in case of the Newtonian heating parameter, shrunk sheet produced higher thermal boundary layer thickness, and the stretched sheet had $6.4 \%$ more heat transfer rate than that of shrunk sheet. Prospectively, it is an interesting question to investigate the similar flow behaviour of the micropolar fluid flow over a wedge in the porous media.

\section{Acknowledgment}

This research is financially supported by, Spk:319299-Ver 1, 6353 Curtin University-SCI \& ENG Department of Mathematics and Statistics/CIPRS. Moreover, there is no conflict of interest in this presented research.

\section{References}

[1] A.C. Eringen, Theory of micropolar fluids, J. Math. Mech. 16(1966), 1-18. 
[2] T. Ariman, A. S. Cakmak, Some basic viscous flows in micropolar fluids, Rheol. Acta 7(1968), 236-242.

[3] Y. Kazakia, T. Ariman, Heat-conducting micropolar fluids, Rheol. Acta 10(1971), 319-325.

[4] A.C. Eringen, Theory of Thermomicrofluids, J. Math. Anal. Appl., 38(1972), 480-496.

[5] A.J. Willson, Boundary layers in micropolar liquids, Math. Proc. Camb. Philos. Soc. 67(1970), 496-476.

[6] T. Ariman, M. A. Turk, N. D. Sylvester, On steady and pulsatile flow of blood, J. Appl. Mech. 41(1974), 1-7.

[7] N. P.Migun, Experimental method of determining parameters characterizing the microstructure of micropolar liquids, J. Eng. Phy. 41(1981), 832-835.

[8] V. L. Kolpashchikov, N. P. Migun, P. P. Prokhorenko, Experimental determination of material micropolar fluid constants, Int. J. Eng. Sci. 21(1983) 405-411.

[9] N. T. Eldabe, M. E. Ouaf, Chebyshev finite difference method for heat and mass transfer in a hydromagnetic flow of a micropolar fluid past a stretching surface with Ohmic heating and viscous dissipation, Appl. Math. Comput. 177(2006), 561-571.

[10] A. Ishak, R. Nazar, I. Pop, Boundary-layer flow of a micropolar fluid on a continuously moving or fixed permeable surface, Int. J. Heat Mass Transf. 50(2007), 4743-4748.

[11] A. Ishak, Thermal boundary layer flow over a stretching sheet in a micropolar fluid with radiation effect, Meccanica. 46(2010), 367-373.

[12] N. Bachok, A. Ishak, R. Nazar, Flow and heat transfer over an unsteady stretching sheet in a micropolar fluid, Meccanica. 46(2011) 935-942.

[13] L. Zheng, J. Niu, X. Zhang, L. Ma, Dual solutions for flow and radiative heat transfer of a micropolar fluid over stretching/shrinking sheet, Int. J. Heat Mass Transf. 55(2012), 7577-7586.

[14] H. H. Sherief, M. S. Faltas, El-Sapa. Shreen, Pipe flow of magneto-micropolar fluids with slip, Can. J. Phy. 95(2017), 885-893.

[15] M.A. Ezzat, ElâĂ ̌̌Sapa. Shreen, State space approach to magnetohydrodynamic flow of perfectly conducting micropolar fluid with stretch, Int. J. Numer. Meth. Fl. 70(2012), 114-134.

[16] R. C. Chaudhary, P. Jain, Combined heat and mass transfer in magnetoâĂŘmicropolar fluid flow from radiate surface with variable permeability in slipâ̆̆̌̆flow regime, ZAMMâĂ ̌̌J. Appl. Math. Mech. 87(2007) 549-563.

[17] R. A. Mohamed, S. M. Abo-Dahab, Influence of chemical reaction and thermal radiation on the heat and mass transfer in MHD micropolar flow over a vertical moving porous plate in a porous medium with heat generation, Int. J. Therm. Sci. 48(2009), 1800-1813.

[18] A. J. Chamkha, R. A. Mohamed, S. E. Ahmed, Unsteady MHD natural convection from a heated vertical porous plate in a micropolar fluid with Joule heating, chemical reaction and radiation effects, Meccanica. 46(2011), 399-411.

[19] A. A. Bakr, Effects of chemical reaction on MHD free convection and mass transfer flow of a micropolar fluid with oscillatory plate velocity and constant heat source in a rotating frame of reference, Commun. Nonlinear Sci. Numer. Simul. 16(2011) 698-710.

[20] T. Hayat, S. A. Shehzad, M. Qasim, Mixed convection flow of a micropolar fluid with radiation and chemical reaction, Int. J. Numer. Meth. Fl. 67(2011), 1418-1436.

[21] D. Pal, B. Talukdar, I. S. Shivakumara, K. Vajravelu, Effects of hall current and chemical reaction on oscillatory mixed convection-radiation of a micropolar fluid in a rotating system, Chem. Eng. Commun. 199(2012), 943965. 
[22] L. Wu, A slip model for rarefied gas flows at arbitrary Knudsen number, Appl. Phys. Letter, 93(2008), 253103.

[23] T. Fang, S. Yao, J. Zhang, A. Aziz, Viscous flow over a shrinking sheet with a second order slip flow model, Commun. Nonlinear Sci. Num. Simul., 15(2010), 1831-1842.

[24] M. M. Nandeppanavar, K. Vajravelu, M. S. Abel, M. N. Siddalingappa, Second order slip flow and heat transfer over a stretching sheet with non-linear Navier boundary condition, Int. J. Therm. Sci. 58(2012), 143-150.

[25] G. Singh, A.J. Chamkha, Dual solutions for second-order slip flow and heat transfer on a vertical permeable shrinking sheet, Ain Shams Eng. J. 4(2013), 911-917.

[26] M. Turkyilmazoglu, Heat and mass transfer of MHD second order slip flow, Comput. Fl. 71(2013), 426-434.

[27] N.C. Rosca, I. Pop, Boundary layer flow past a permeable shrinking sheet in a micropolar fluid with a second order slip flow model, Eur. J. Mech. B-Fluid, 48(2014), 115-122.

[28] R. Sharma, A. Ishak, I. Pop, Stagnation point flow of a micropolar fluid over a stretching/shrinking sheet with second-order velocity slip, J. Aerospace Eng., 29(2016), 04016025.

[29] W. Ibrahim, MHD boundary layer flow and heat transfer of micropolar fluid past a stretching sheet with second order slip, J. Brazilian Soc. Mech. Sci. Eng., 39(2017), 791-799.

[30] J. H. Merkin, Natural-convection boundary-layer flow on a vertical surface with Newtonian heating, Int. J. Heat Fl. Flow. 15(1994), 392-398.

[31] G. Ahmadi, Self-similar solution of imcompressible micropolar boundary layer flow over a semi-infinite plate, Int. J. Eng. Sci., 14(1976), 639-646.

[32] K. Bhattacharyya, S. Mukhopadhyay, G.C. Layek, I. Pop, Effects of thermal radiation on micropolar fluid flow and heat transfer over a porous shrinking sheet, Int. J. Heat Mass Transf. 55(2012), 2945-2952.

[33] S.J. Liao, "The proposed homotopy analysis technique for the solution of nonlinear problems," Ph.D. thesis, Shanghai Jiao Tong University. 1992.

[34] S.J. Liao, Beyond Perturbation: Introduction to the Homotopy Analysis Method, Chapman and Hall: Boca Raton, 2003.

[35] L.F. Shampine, I. Gladwell, S. Thompson, Solving ODEs with MATLAB, Cambridge University Press, 2003.

[36] L.F. Shampine, M.W. Reichelt, J. Kierzenka, Solving boundary value problems for ordinary differential equations in Matlab with bvp4c, 2010. 